This paper has been accepted for publication in 2017 IEEE International Conference on Communications Workshops (ICC Workshops) (C) 2017 IEEE. Personal use of this material is permitted. Permission from IEEE must be obtained for all other uses, in any current or future media, including reprinting/republishing this material for advertising or promotional purposes, creating new collective works, for resale or redistribution to servers or lists, or reuse of any copyrighted component of this work in other works. DOI: 10.1109/ICCW.2017.7962771

\title{
Towards SDN/NFV-enabled satellite ground segment systems: End-to-End Traffic Engineering Use Case
}

\author{
R. Ferrus ${ }^{1)}$, O. Sallent ${ }^{1)}$, T. Ahmed ${ }^{2)}$, R. Fedrizzi ${ }^{3)}$ \\ ${ }^{1)}$ Universitat Politècnica de Catalunya, Spain; ${ }^{2)}$ CNRS-LaBRI, University of Bordeaux, France; ${ }^{3)}$ Centre for Research and \\ Telecommunication Experimentation for Networked communities, Italy
}

\begin{abstract}
Key features of satellite communications such as wide-scale coverage, broadcast/multicast support and high availability, together with significant amounts of new satellite capacity coming online, anticipate new opportunities for satellite communications services as an integral part within upcoming 5G systems. To materialize these opportunities, satellite communications services have to be provisioned and operated in a more flexible, agile and cost-effective manner than done today. In this context, this paper firstly describes the architecture of a satellite ground segment system that builds on the introduction of Software Defined Networking (SDN) and Network Function Virtualization (NFV) technologies and then examines a use case for the realization of End-to-End Traffic Engineering in a combined terrestrial-satellite network used for mobile backhauling.
\end{abstract}

Keywords-Satellite network; Network Function Virtualization; Software-Defined Networking; Satellite gateway virtualization; Combined satellite-terrestrial networks; service orchestration

\section{INTRODUCTION}

The role that satellite communications can play in the forthcoming $5 \mathrm{G}$ ecosystem is being revisited [1][2][3]. Ubiquitous broadband connectivity, extended to rural and lowdensity areas as well as long-haul transportation (e.g. aircraft, trains), are recognized as key requirements for $5 \mathrm{G}$ [4]. To fulfil these requirements, the role of satellite networks is fundamental to reach those areas where the terrestrial service is limited or simply not available as well as for the delivery of services that can be more efficiently supported through satellite communications (e.g. multimedia distribution) or benefit from higher dependability (e.g. critical communications). Technological advances in the satellite domain such as the use of High Throughput Satellites (HTS) in Geostationary Earth Orbit (GEO) is changing the way that capacity is being brought to the market, considerably reducing the price per bit. By 2020 2025 it is expected that there will be over 100 HTS systems in orbit, delivering Terabits of connectivity across the world using $\mathrm{Ku}$ - and $\mathrm{Ka}$ - bands [5]. Likewise, a range of disruptive initiatives envisioning the use of non-GEO constellations with a large number of low-cost micro-satellites [6] might come to fruition in the forthcoming years, anticipating a further reduction in the cost together with performance improvements in terms of latency. This paves the way for a more central role of a satellite component as an integral part of next generation networks. Remarkably, a requirement for next generation 3GPP systems to provide services using satellite access has been included within the normative Stage 1 requirements [7].

In this context, the evolution of satellite ground segment systems (e.g. satellite gateways and terminals) from today's rather closed solutions towards more open architectures based on Software Defined Networking (SDN) and Network Function Virtualization (NFV) technologies arises as a necessary step [8][9], not only to bring into the satellite domain the benefits associated with the advances in network softwarisation technologies that are being consolidated within the $5 \mathrm{G}$ landscape, but also to greatly facilitate the seamless integration and operation of combined satellite and terrestrial networks [10]. In particular, terrestrial 5G systems are widely embracing SDN technologies for enabling a unified, vendor-neutral control and management of networking functions. Therefore, satellite networks shall be outfitted with a set of control and management functions and interfaces (API and/or network protocols) compatible with the mainstream SDN architectures and technologies being adopted in $5 \mathrm{G}$ in order to realize a full Endto-End (E2E) networking concept where the whole satelliteterrestrial network behaviour can be programmed in a consistent and interoperable manner.

This paper examines a use case for the realization of E2E Traffic Engineering (TE) in a combined terrestrial-satellite network embracing SDN technologies. In particular, the presented case is that of a mobile network where a SDN-capable satellite network is integrated as part of the backhaul infrastructure so that the end-to-end paths across the satellite and terrestrial components can be centrally computed and rearranged dynamically at flow-level granularity in front of link congestion and failure events. To that end, the main contributions of this paper are the proposal to abstract the satellite component as an OpenFlow (OF) switch for its integration within the mobile backhaul and the elaboration of illustrative TE workflows to validate the proposed approach. The rest of this paper is organized as follows. Section II outlines the main architectural traits of a satellite ground segment system architecture grounded on SDN/NFV principles and technologies. Section III and IV provide baseline considerations regarding, respectively, the support of TE mechanisms in SDNbased networks and the introduction of SDN solutions in mobile networks. On this basis, Section V presents a functional view for the integration of the SDN-capable satellite network within the mobile network and establishes a reference network topology for the development of the TE workflows, which are detailed in 
Section VI. Finally, concluding remarks are drawn in Section VII.

\section{SDN/NFV-ENABLED SATELLITE GROUND SEGMENT SYSTEMS}

Our view of a Virtualized Satellite Network (VSN) is that of a satellite network in which most of their functions are supplied as software components (i.e. Virtual Network Functions [VNFs]) and in which control and management capabilities are supported and exposed through SDN-based interfaces. An illustrative view of the VSN concept is given in Fig.1. By Satellite Baseband Gateway - Physical Network Function (SBGPNF) we denote the components of the VSN that could be still supplied in specialized hardware, which is likely to support the physical layer functionality of the satellite gateway and be located at teleport locations. On the virtualized part, which would run in a NFV infrastructure that could be distributed among teleports and centralized locations, the illustrated VSN consists of VNFs that handle the link layer data plane functions of the satellite gateway (denoted by SBG-VNF in Fig.1), a VNF for handling the control plane functions of the satellite network (denoted by VSN Network Control Centre [VSN NCC] in line with terminology specified in [11][12]), a VNF for the management plane functions (denoted by VSN Network Management System / Element Management System [VSN NMS/EMS]), and a set of VNFs to handle satellite network functions such as Performance Enhancing Proxies (PEPs) (denoted generically by SNF-VNF).

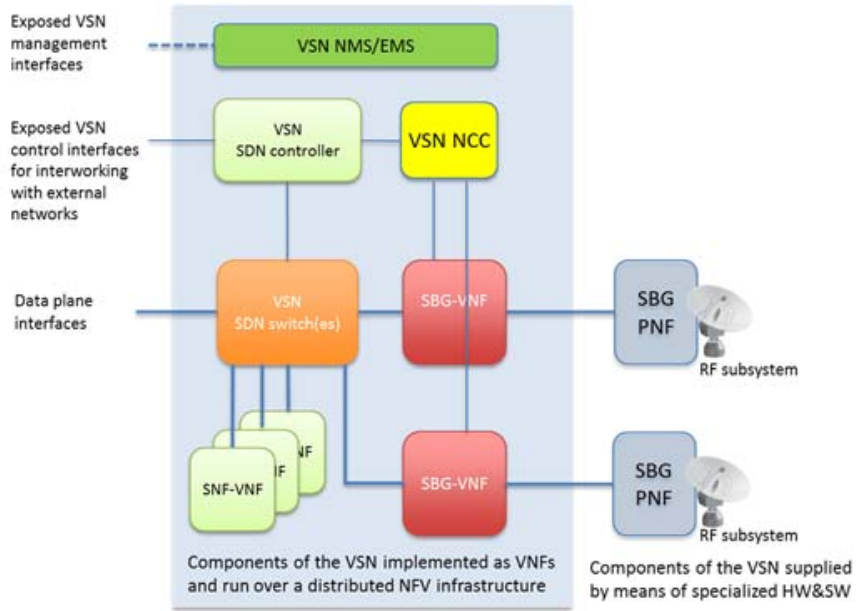

Fig. 1. Illustration of a multi-gateway VSN with integrated SDN controller that exposes control interfaces for interworking with an external network

In addition, the VSN also comprises a number of SDN switches and a SDN controller (i.e. these could be virtualized elements deployed as part of the VSN). The SDN switches are used to support the internal L3/L2 data plane forwarding functions across the components of the VSN as well as for the interworking with external networks (e.g. these SDN switches can add/drop VLAN tags/MPLS headers, enforce differentiate QoS treatments, etc., both for internal or for external interworking purposes). The VSN SDN controller is used (1) to control the operation of the SDN switches, (2) to support dynamic activation of the data transmission services (e.g. satellite bearer services [11]) through an interface with the VSN NCC functions, and (3) to expose a control interface for interworking with external networks. It's worth noting that Fig.1 only intends to provide an illustrative example, introducing the main features of the VSN that are later on referred to in the description of the workflows. But other variants are possible depending on the types and characteristics of the VNFs that could be eventually conceived / developed to satisfy whatever specific needs (e.g., market demand, scalability issues, further split of functionality, etc.). Additional details on the VSN architecture and on the components and mechanisms for the dynamic instantiation of several, tenant-customized VSNs on top of a common SDN/NFV-enabled satellite ground segment system are given in [13].

\section{EVOLUTION TOWARDS SDN-BASED TE}

TE mechanisms are used to optimize the performance of a data network by dynamically analysing, predicting, and regulating the behaviour of the traffic across the network [14]. As of today, multiple approaches for TE have been specified to different extent for MPLS networks, which is still the dominant data plane technology for delivering reliable Quality of Service (QoS) on IP-based networks through QoS-based routing and other advanced capabilities. Existing MPLS-TE approaches can be divided into distributed and centralized approaches according to the way paths are set up in the network [15]. In distributed approaches, topology attributes are disseminated via extensions of existing routing protocols such as Open Shortest Path First (OSPF)-TE or Intermediate System to Intermediate System (ISIS)-TE. Then, the head-end MPLS node of each individual Label Switched Path (LSP) is the one in charge of computing the path for its LSPs based on topology and constraints learned through the routing protocol. Signalling of the LSPs through the network is done using protocols such as Reservation Protocol (RSVP)TE or Label Distribution Protocol (LDP). The drawback of this approach is the required signalling through the network, which, together with the need to store LSP state on all routers, can represent a scalability problem in large networks. Therefore, while the MPLS data plane is fairly simple, the control planes associated with MPLS-TE are rather complicated. On the other hand, in centralized approaches, the LSP calculation is often performed offline in a management system and the LSP configuration is then pushed into the nodes through Operation and Maintenance procedures. The advantage of a centralized approach is that it can consider application requirements more easily than a distributed path computation in the network nodes. Central path calculation also has the ability to compute more optimal paths, based on global knowledge, which results in better network resource utilization. The drawback of the traditional approach of centralized offline calculation is a lack of agility and the need for complex and expensive management software. It also involves a high degree of manual work to prepare the necessary input for path calculation. This results in high cost and slow response times. In the context of satellite communications, support of MPLS technologies, and by extension MPLS-TE, has been addressed in ETSI TC SES specifications [16][17], which describe the functional adaptations that are needed to enable Broadband Satellite Multimedia (BSM) networks to support MPLS efficiently and in a standardized manner. Indeed, the main motivation of the ETSI specifications is to achieve MPLS compatibility between satellite networks and terrestrial networks, for example to 
provide extensions and interconnectivity to terrestrial MPLS networks or to provide a satellite back-up for terrestrial MPLS networks.

With the advent of SDN principles (i.e. separation of the control plane from the physical network for visibility, centralization and programmability with granular control), new solutions that can overcome the limitations of existing MPLSTE solutions become feasible [15][18]. In particular, an SDNbased approach has the following key characteristics: (1) path calculation can be done in a centralized controller and (2) the controller supports various southbound interfaces toward the network elements for configuring the calculated path in the data plane. Indeed, the unique features of SDN enable more sophisticated TE techniques that exploit the global network view, status, and flow patterns/characteristics available for better traffic control and management, including mechanisms for flow management, fault tolerance, topology update, and traffic analysis [19]. It's worth noting that the evolution of MPLS-TE solutions towards supporting centralized path computation already started with the Path Computation Element (PCE) protocol [20], an IETF standard that works in MPLS networks and partially removes the control plane from head-end routers to define network paths. While PCE can leverage the installed base of MPLS equipment and technology by migrating only the path computation component to a centralized role and leaving the remaining components (discovery, database distribution and path setup) within the MPLS nodes, it is mainly been considered as an intermediate stage in a smooth migration plan towards fullfledged SDN networks [21].

\section{INTRODUCTION OF SDN TECHNOLOGIES WITHIN NEXT GENERATION MOBILE NETWORKS}

Several proposals exist for adopting SDN concepts in mobile network architectures [22][23]. In general terms, an illustrative view of a SDN-based mobile that uses SDN-enabled transport from the Radio Access Network (RAN) nodes (e.g. Base Stations [BSs]) all the way through the backhaul to the core network is depicted in Fig.2. Though this architecture is contextualized for LTE technology, this vision is claimed to be generic and not constrained by the specifics of the LTE standard. As depicted in Fig.2, mobile core network control functions (e.g. Mobility Management Entity [MME] and Serving / Packet Data Network [PDN] gateways (S/P - GW) functional elements in LTE Evolved Packet Core) together with specific TE functions for the transport network are realized as applications running on top of a SDN controller (represented here as a single functional entity but likely to follow a hierarchical structure of controllers). This SDN controller is responsible for managing the Network Elements (NE) that provide the packet switching and forwarding capabilities within the transport network. In this respect, the underlying transport network infrastructure may involve a number of different physical network equipment, or forwarding devices such as routers, switches, virtual switches, to name a few. In a software-defined network, such devices are often represented as basic forwarding hardware accessible via an open interface at an abstraction layer, as the control logic and algorithms are off-loaded to a SDN controller. Such forwarding devices are frequently referred to, in SDN terminology, simply as "switches". This SDN controller serves as the brain of the transport network and calculates and installs rules into the NE, down to the BSs. This architecture would enable dynamic control of individual traffic flows, allowing fine-grained TE across the whole network in the most convenient way (a flow is commonly defined as a stream of data that is identifiable by some criteria such as common packet header values, relative time position or frequency and is acted on and/or forwarded based on a common set of rules within the bounds of a connection [25]). The centralized control and programmability capability of SDN-based TE deployed across all the different technologies and networking layers co-existing in the mobile backhaul network would greatly simplify its operation and make network resource management more flexible and efficient (e.g. through capacity-aware end-to-end path computation). While until recently the SDN scope has been focused on the packetoriented Layers 2 and 3 (e.g. Ethernet, IP/MPLS), different extensions are underway to support abstractions necessary in mobile networks [23], manage optical transmission devices (Transport SDN [22][24]) and wireless transport devices (Wireless Transport Networks [26]).

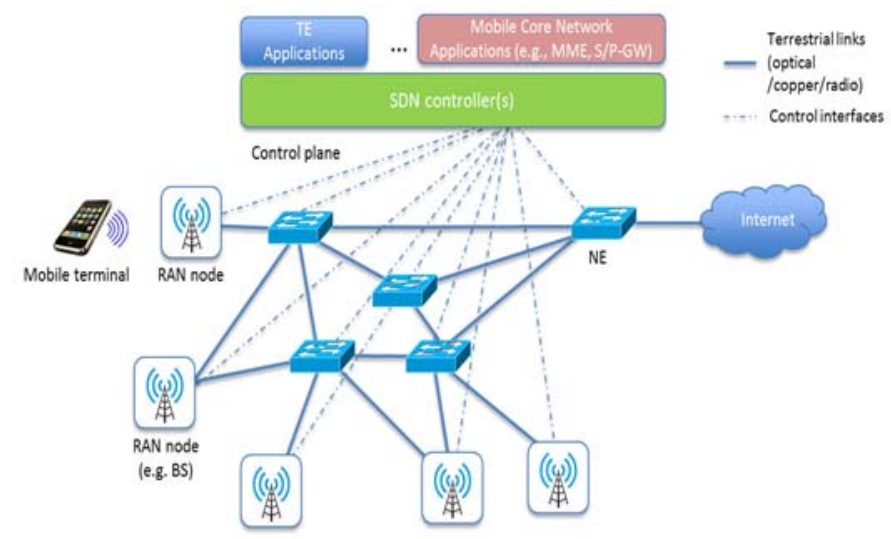

Fig. 2. Illustrative view of a SDN-based mobile network

One central protocol in SDN architectures is OpenFlow (OF) [27]. In an OF-based SDN architecture, the forwarding device, or OF switch, contains one or more flow tables and an abstraction layer that securely communicates with a controller via OF protocol. Flow tables consist of flow entries, each of which determines how packets belonging to a flow will be processed and forwarded. Flow entries typically consist of: (1) match fields, or matching rules, used to match incoming packets; match fields may contain information found in the packet header (Layer 2-7 information), ingress port, and metadata; (2) counters, used to collect statistics for the particular flow, such as number of received packets, number of bytes and duration of the flow; and (3) a set of instructions, or actions, to be applied upon a match; they dictate how to handle matching packets. The full specification is available in [28]. Indeed, OF is an extensible protocol, providing mechanisms for SDN programmers to define additional protocol elements (e.g., new match fields, actions, port properties, etc.) to address new network technologies and behaviours.

\section{PROPOSED INTEGRATION APPROACH FOR E2E TRAFFIC ENGINEERING: FUNCTIONAL VIEW}

A key feature of the VSN concept presented in Section II is the exposition of control and management capabilities through an SDN-based interface. Such an interface would allow the 
Mobile Network Operator (MNO) to integrate the VSN within the mobile backhaul network and to operate in a coordinated and consistent manner the whole end-to-end chain from network controllers that could be located in the MNO's infrastructure.

Building on the general view of a SDN-based mobile network previously depicted in Fig.2, Fig.3 depicts the functional view of the proposed integration approach, which is founded on two main concepts:

-Abstraction of the overall VSN as a SDN-capable "switch". In particular, the OF switch abstraction model [28] is considered to model the operation of the VSN as seen from an external controller entity. Internally, the VSN comprises the set of Satellite Terminals (ST) and gateways (SBG-PNF + SBG VNFs) that are interconnected with the outside world through standard switching/routing functions used to provide L3 services such as IP/MPLS or L2 services such as Carrier Ethernet Services (CES). It is assumed that the VSN SDN controller inside the VSN orchestrates the operation of both switching/routing functions at the edges and the activation/deactivation of the satellite connections and its configuration through the VSN NCC. This SDN controller is the one that exposes an abstract view of the VSN through the OF interface.

-Use of SDN-based TE applications, with a central Path Computation Engine (PCE) that support the operation of the Mobile Core Network (MCN) for traffic management within the backhaul transport network. It is assumed that the overall transport network is managed as a single logical forwarding domain and that, inside the forwarding domain, a MNO's SDN controller makes the forwarding decisions. As depicted in Fig.3, all SDN-capable L2/L3 NEs are connected to the MNO's SDN network controller through OF interfaces, including the "VSN switch". In this way, SDN-based TE mechanisms can seamlessly span the whole network. For the terrestrial connection, no specific technology is assumed rather than considering that traffic flows can also be managed through SDN features.

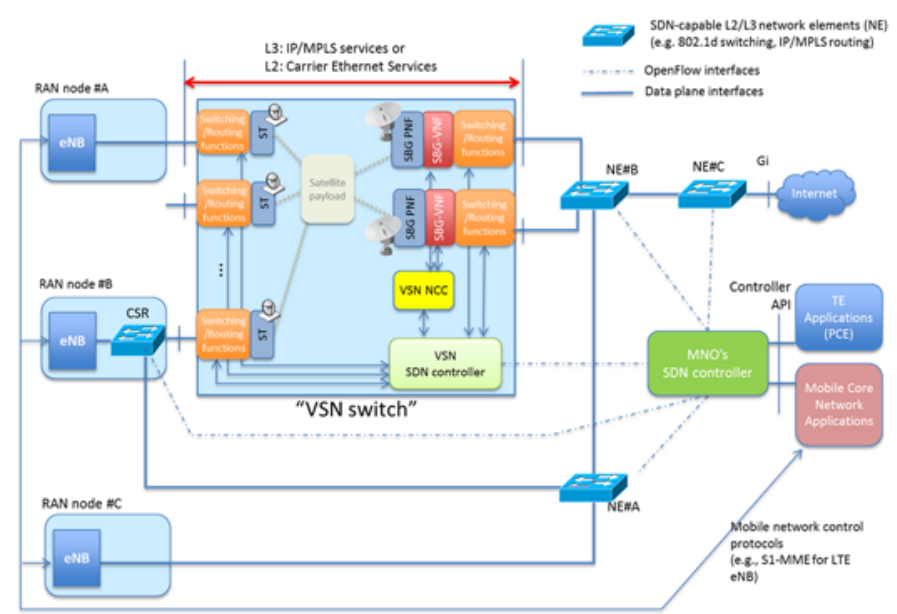

Fig. 3. Functional view and illustrative network topology considered for the development of the TE procedures

In order to raise different considerations with regard to the operation of TE procedures, the illustrative network topology depicted in Fig. 3 considers three RAN nodes, one connected to the transport network only by terrestrial means (RAN node\#C), another connected only through the VSN (RAN node\#A) and a third one (RAN node\#B) connected to both a terrestrial connection and a satellite connection through a SDN-capable Cell Switch Router (CSR). This third case is used to illustrate the realisation of TE mechanisms for multi-path optimization [30]. With respect to the terrestrial part of the transport network, three NE are included in the reference network topology, two of them acting as internal aggregation/core nodes within the transport network (i.e. NE\#A and NE\#B) and the third one (i.e. NE\#C) providing the interconnection with the external networks (e.g. Internet) through a conventional 3GPP Gi interface. Of note is that, in addition to OF interfaces for controlling the forwarding function of the transport network, other control interfaces are likely to be in place in the overall setting for other purposes, such as the 3GPP S1-MME interface between the Mobile Core Network (MCN) applications and the eNBs within the RAN nodes to manage the activation/deactivation of radio access bearers in the eNB for the served mobile terminals.

\section{PROPOSED INTEGRATION APPROACH FOR E2E TRAFFIC ENGINEERING: ILLUSTRATIVE E2E TE WORKFLOWS}

Two illustrative workflows are developed in the following sections to validate the proposed integration approach. The first one shows the activation of a traffic flow through the satelliteterrestrial network to enforce a mobile network bearer (e.g. socalled EPS bearer in the context of LTE) that can benefit from optimal path computation. The second workflow shows the modification of an already established flow as a reaction to a congestion/failure situation in one link within the transport network.

\section{A. Flow activation with optimal path computation}

Based on the network topology depicted in Fig.3, a message chart with the operation of a path computation mechanism for multi-path satellite-terrestrial traffic optimisation is provided in Fig.4. In particular, the provided workflow covers the case of the establishment of a dedicated EPS Bearer Service that relies on the TE path computation mechanism to activate the traffic path between the RAN node and the external network reachable through NE\#C considering the characteristics of the EPS bearer and the load conditions across the whole network. It is assumed that the SDN controller has a global view of network topology which can be represented by means of a graph including all links between OF switches (the links could be found by e.g. leveraging protocols such as LLDP (802.1AB), which is used by network devices to advertise their identity, capabilities, and neighbours). Details of the different steps depicted in Fig. 4 are given in the following:

Step 1: Monitoring of the SDN forwarding elements within the domain, including the CSR, "VSN switch" and NEs. TE needs granular real-time monitoring information to compute the most efficient routing decisions. Solutions such as the one described in [31] allows for an OF controller to have accurate monitoring of per-flow throughput, packet loss and delay metrics in order to aid TE. In this respect, while a flow is active, the controller and the SDN forwarding element can exchange messages concerning the state of the flow. 
Step 2: As a result of the activation of a new service (e.g., HD video-streaming service) by a mobile terminal connected in RAN node\#B, the MCN decides to establish a new dedicated EPS bearer to support that service. The activation of the dedicated EPS bearer requires the activation of a flow with QoS guarantees across the transport network. The two edge nodes of the EPS bearer are the RAN node\#B, where the UE is assumed connected, and the NE\#C, which serves as the gateway to the external network.

Step 3: The MCN request to the TE application the computation of the best path between RAN node\#B and NE\#C. QoS attributes of the EPS bearer are indicated (e.g., Guaranteed Bit Rate).

Step 4: Based on the (1) network topology knowledge, (2) the network monitoring information and QoS attributes of flow, the TE application can compute the most appropriate path. Different algorithms could be supported here, including graph searching algorithms for path finding and algorithms for path selection depending on policies with respective of traffic engineering or service quality, such as calculating the shortest path forwarding based on a consistent view of network state or provision application-aware routing [32]. Anyway, let's consider that the outcome of this decision is that a path through the VSN is chosen for this flow.

Step 5: Flow entries are installed to OF switches along the path by the MNO's SDN controller to make sure traffics between RAN node\#B and NE\#C are forwarded along the selected path. Step 6: The MCN gets the path establishment response.

Step 7: The EPS bearer activation at the radio layer takes places, involving the interaction between the MCN functions and the eNB within RAN node\#B.

Step 8: The data plane for the dedicated EPS bearer gets live and traffic follows the selected path through the VSN.

The above workflow assumes that the path is established to support a single EPS bearer. However, the same approach would be used in case of deciding the best path for traffic aggregates with common QoS requirements. This is well supported in OF by just establishing the corresponding matching conditions (e.g., IP prefixes to identify a traffic aggregate in front of particular IP addresses of the individual flows).

\section{B. Flow update to overcome congestion/failures}

Fig. 5 shows how the failure of a path, or simply the congestion of a path that could cause QoS degradation, could be handled within the proposed integration approach. In particular, the message chart depicted in Fig.5 is a TE mechanism that will update an already established flow in order to overcome a congestion/failure event. Details of the different steps depicted in Fig. 5 are given in the following:

Step 1: The starting point considers that traffic from/to RAN node \#B and from/to RAN node\#C, called Traffic B and Traffic $C$ respectively, are both flowing through NE\#A, NE\#B and NE\#C. This could be assumed to be the optimal traffic path for a moderate traffic load scenario.

Step 2: Monitoring of the SDN forwarding elements is conducted by the MNO's SDN controller, as described in the previous workflow.
Step 3: An event that puts at risk the QoS of the established flows occurs. This could be, for example, a considerable traffic increase in RAN node $\mathrm{CC}$ at certain time of the day that overloads the link among NE\#A and NE\#B, which is shared by Traffic B and Traffic C.

Step 4: The TE application detects the congestion situation. For example, the TE application could have set a high utilization threshold of 60 percent and low utilization threshold of 20 percent for the traffic load on the shared link. If this high threshold is exceeded, high utilization is observed and e.g. a part of Traffic B could be switched to pass through the VSN.

Step 5: Flow entries are installed to OF switches along the path by the MNO's SDN controller to re-route part of the traffic B through the satellite connection.

Step 6: While the path for traffic $\mathrm{C}$ remains unchanged, now part of traffic $\mathrm{B}$ is served through the VSN, reducing congestion in the link between NE\#A and NE\#B.

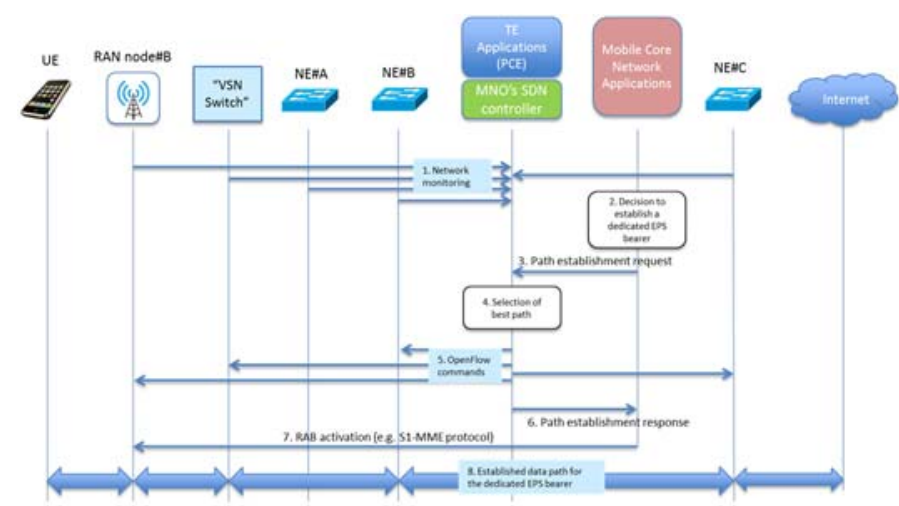

Fig. 4. Flow activation with optimal path computation

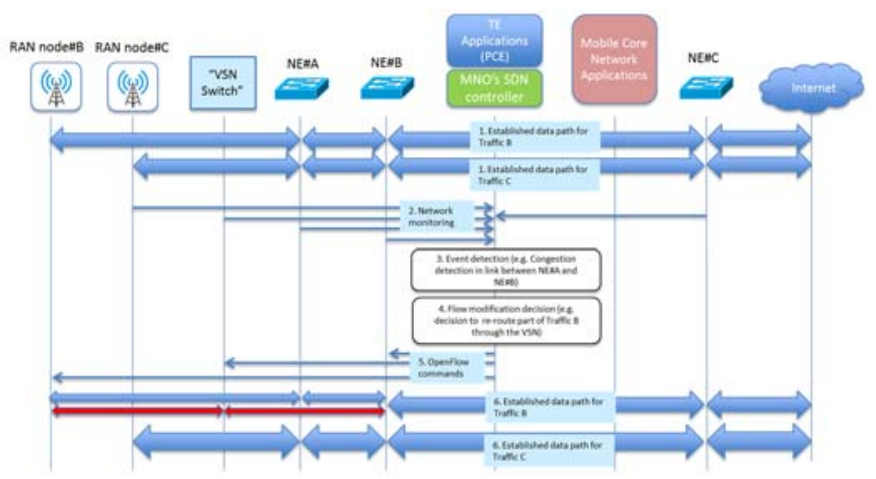

Fig. 5. Flow update to overcome congestion/failures

Flow updates can also be driven by connection protection in case of failure. Indeed, path protection and network recovery from failure are critical aspects of TE. While these aspects are well-understood in conventional MPLS/IP networks, work is still needed to mature these concepts in the context of SDN networks [33].

\section{CONCLUDING REMARKS}

The adoption of SDN and NFV technologies into the satellite domain is seen as a key facilitator to enhance the delivery of satellite communications services and achieve a better integration of the satellite component within the $5 \mathrm{G}$ ecosystem. 
In particular, we advocate for satellite networks to be outfitted with a set of control and management functions and interfaces compatible with the mainstream SDN architectures and technologies being adopted in $5 \mathrm{G}$ in order to realize a full Endto-End (E2E) networking concept where the whole satelliteterrestrial network behaviour can be programmed in a consistent and interoperable manner.

On this basis, this paper has developed a use case for the realization of E2E Traffic Engineering (TE) in a mobile network where a SDN-capable satellite network is integrated as part of the backhaul infrastructure. To that end, we've proposed to abstract the satellite component as an OF switch for its integration within the mobile backhaul and we've elaborated two central TE workflows to validate the proposed approach. In particular, one workflow illustrates how the activation of a traffic flow across the satellite and terrestrial components can be conducted by a SDN-based TE application for optimal path computation. The second workflow shows the modification of an already established flow as a reaction to a congestion/failure situation in one link within the transport network.

\section{ACKNOWLEDGMENT}

Research leading to these results has received funding from the European Union's H2020 Research and Innovation Programme (H2020-ICT-2014-1) under the Grant Agreement H2020-ICT-644843.

\section{REFERENCES}

[1] NetWorld2020's - SatCom WG The role of satellites in 5G, Version 5 31th July 2014

[2] Sacchi, C.; Bhasin, K.; Kadowaki, N.; Vong, F., "Toward the "space 2.0" Era [Guest Editorial]," Communications Magazine, IEEE, vol.53, no.3, pp.16,17, March 2015

[3] Marius Corici et al., "Assessing satellite-terrestrial integration opportunities in the 5G environment", September 2016. Available online at $\quad$ https://artes.esa.int/sites/default/files/Whitepaper\%20$\% 20$ Satellite $5 \mathrm{G} \% 20$ final.pdf

[4] 5G Vision - The 5G Infrastructure Public Private Partnership: the next generation of communication networks and services

[5] "Satellite: An Integral Part of the 5G Ecosystem", ESOA, https://www.esoa.net/cmsdata/positions/5G\%20infographic\%20final_1.pdf

[6] ARTES programme, "ESA announces dedicated support for the development of megaconstellations", Last updated July 2015. Available online at https://artes.esa.int/news/esa-announces-dedicated-supportdevelopment-megaconstellations

[7] 3GPP TS 22.261 V0.1.1 (2016-08), "Service requirements for next generation new services and markets; Stage 1 (Release 15)", August 2016

[8] H2020 VITAL research project website at http://www.ict-vital.eu/

[9] Bertaux L., Medjiah S., Berthou P., Abdellatif S., Hakiri A., Gelard P., "Software Defined Networking and Virtualization for Broadband Satellite Networks". IEEE Communications Magazine, March 2015

[10] R. Ferrús, H. Koumaras, O. Sallent, G. Agapiou, T. Rasheed, M.-A. Kourtis, C. Boustie, P. Gelard, T. Ahmed, SDN/NFV-enabled satellite communications networks: Opportunities, scenarios and challenges, Physical Communication, November 2015

[11] ETSI TR 101984 V1.2.1, "Satellite Earth Stations and Systems (SES); Broadband Satellite Multimedia (BSM); Services and architectures", December 2007

[12] ETSI TR 102603 V1.1.1, "Satellite Earth Stations and Systems (SES); Broadband Satellite Multimedia (BSM); Connection Control Protocol (C2P) for DVB-RCS; Background Information", January 2009

[13] R. Ferrús, H. Koumaras, O. Sallent, T. Rasheed, E. Duros, R. Riggio, N. Kuhn, P. Gelard, T. Ahmed, "On the virtualization and Dynamic
Orchestration of Satellite Communication Services", In Proceedings of 84th IEEE Vehicular Technology Conference (VTC'16-Fall), Montreal, Canada, September 2016

[14] IETF RFC 2702: "Requirements for Traffic Engineering over MPLS".

[15] Ericsson White paper, "Wide area network Traffic Engineering", Uen 284 23-3249, December 2014

[16] ETSI TS 102 856-1 V1.1.1, "Multi-Protocol Label Switching (MPLS) interworking over satellite; Part 1: MPLS-based Functional Architecture", July 2011

[17] ETSI TS 102 856-2 V1.1.1, "Multi-Protocol Label Switching (MPLS) interworking over satellite; Part 2: Negotiation and management of MPLS labels and MPLS signalling with attached networks", July 2011

[18] Ali Reza Sharafat, Saurav Das, Guru Parulkar, and Nick McKeown. 2011. MPLS-TE and MPLS VPNS with openflow. In Proceedings of the ACM SIGCOMM 2011 conference (SIGCOMM '11). ACM, New York, NY, USA, 452-453. DOI=http://dx.doi.org/10.1145/2018436.2018516

[19] Ian F. Akyildiz, Ahyoung Lee, Pu Wang, Min Luo, Wu Chou, "A roadmap for traffic engineering in SDN-OpenFlow networks", Elsevier Computer Networks, Volume 71, 4 October 2014, Pages 1-30, doi:10.1016/j.comnet.2014.06.002

[20] David B. Jacobs, "Path Computation Element primer: Are PCE and SDN connected?", June 2013. Available online at http://searchsdn.techtarget.com/tip/Path-Computation-Element-primerAre-PCE-and-SDN-connected

[21] Metaswitch Networks Whitepaper, "PCE - An Evolutionary Approach To SDN", 2012. Company website: http://networktechnologies.metaswitch.com

[22] Bojic D. at al., "Advanced wireless and optical technologies for small-cell mobile backhaul with dynamic software-defined management". IEEE Communications Magazine 51(9):86-93. 2013.

[23] M. R. Sama, L. M. Contreras, J. Kaippallimalil, I. Akiyoshi, H. Qian and $\mathrm{H}$. Ni, "Software-defined control of the virtualized mobile packet core," IEEE Communications, vol. 53, no. 2, pp. 107-115, Feb. 2015.

[24] Open Networking Foundation, "OpenFlow-enabled Transport SDN", ONF Solution Brief, May 2014

[25] Open Networking Foundation, "SDN Architecture for Transport Networks", ONF TR-522, March 2016

[26] Open Networking Foundation (ONF), "Wireless \& Mobile Working Group". Charter description available online at www. opennetworking. org/images/stories/downloads/working-groups/charterwireless-mobile.pdf

[27] B. A. A. Nunes et al., "A Survey of Software-Defined Networking: Past, Present, and Future of Programmable Networks," in IEEE Communications Surveys \& Tutorials, vol. 16, no. 3, pp. 1617-1634, Third Quarter 2014. doi: 10.1109/SURV.2014.012214.00180

[28] Open Networking Foundation, "OpenFlow Switch Specification Version 1.5.1 ( Protocol version 0x06 )”, ONF TS-025, March 2015

[29] Open Transport Charter within the ONF. Official website at https://www.opennetworking.org/technicalcommunities/areas/specification

[30] MPLS in Mobile Backhaul Networks, Framework and Requirements, Technical Specification, IP/MPLS Forum 20.0.0, IP/MPLS Forum Technical Committee, October 2008

[31] N. L. M. van Adrichem, C. Doerr and F. A. Kuipers, "OpenNetMon: Network monitoring in OpenFlow Software-Defined Networks," 2014 IEEE Network Operations and Management Symposium (NOMS), Krakow, 2014, pp. 1-8. doi: 10.1109/NOMS.2014.6838228

[32] Aricent white paper, "Demystifying routing services in software-defined networking". Available online at http://www.aricent.com/sites/default/files/pdfs/Aricent-DemystifyingRouting-Services-SDN-Whitepaper.pdf

[33] Rashmi Pujar, Icaro Camelo, "Path protection and Failover strategies in SDN networks", Inocybe Technologies", Open Networking Summit, March 2016 